
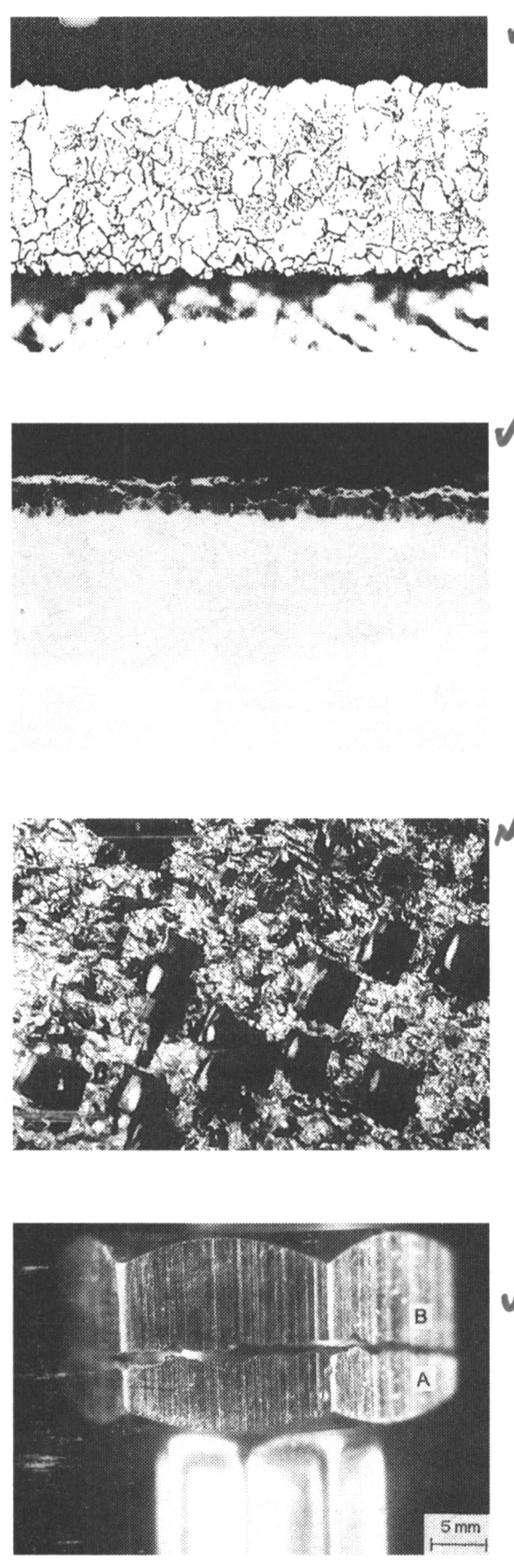

P. Bley

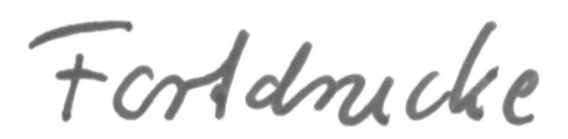

$D H$

Anforderungen der Mikrosystemtechnik an die Materialforschung

The Materials Research Requirements of Microsystems Technology

\section{0}

\section{Kurz, E. Ittlinger}

Ausbildung der Nitrierschichten beim Nitrocarburieren von Ventilen

The Formation of Nitride Layers in the Nitrocarburizing of Automotive Engine Valves

$$
50 \quad 60,-205
$$

\section{E. Favret, F. Povolo, A. Canzian}

Determination of Crystall Orientations in Aluminium by Means of Unidirectional Laser Oblique Illumination (ULOI)

Bestimmung der Kristallorientierungen in Aluminium mittels unidirektioneller Laserschrägeinstrahlung

Schadensanalyse/Failure Analysis

\title{
B. Kuznicka
}

Failure Analysis of a Brass Union Nut of a Flexible Connection Schadensuntersuchung an einer Messing-Überwurfmutter einer beweglichen Verbindung

- Wissenschaftlicher Beirat / Editorial Board

- Mitteilungen / Information 174,215

- Literatur-Notizen / Literature Reviews 205, 225

- Diskusionsforum / Topic of Discussion 


\section{Praktische Metallographie \\ Practical \\ Metallography}

\section{Gründer/Founders}

Günter Petzow

Gerhard Reinacher +

Charlotte Wachau +

Herausgeber/Editor

Prof. Dr. G. Petzow

\section{Schriftleiter}

Editor in charge

Prof. Dr.-Ing. F. Mücklich

Universität des Saarlandes

Lehrstuhl für Funktionswerkstoffe

Postfach 151150

D-66041 Saarbrücken

Telefon: +49/681 302-2048

Telefax: +49/681 302-4876

E-Mail: muecke@matsci.uni-sb.de

\section{Redaktionssekretariat}

Editing Secretary

Dipl.-Ing. S. Mücklich

Bei Gerstnershaus 31a

D-66125 Saarbrücken

Telefon: $+49 / 689771975$

Telefax: $+49 / 6897729810$

E-Mail:

pract.metallog@matsci.uni-sb.de

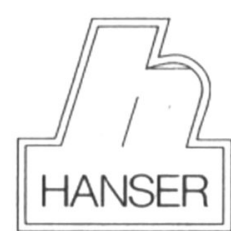

Carl Hanser Verlag, München

\section{Wissenschaftlicher Beirat/Editorial Board}

Dr. C. Bagnall, MCS Associates Inc., Greensburg, PA (USA)

Dr. E. Bischoff, MPI für Metallforschung, Stuttgart

C. Bochert, Wirtz-Buehler GmbH, Düsseldorf

Prof. Dr. H.-E. Bühler, RWTH Aachen

Dr. V. Dietl, Lette Verein Berlin

Dr. G. Elssner, MPI für Metallforschung, Stuttgart

Prof. Dr. H.E. Exner, TU Darmstadt

Prof. Dr. E. Hornbogen, Ruhr-Universität Bochum

Prof. Dr. F. Jeglitsch, Montanuniversität Leoben

Dr. H.-J. Klaar, RWTH Aachen

Prof. Dr. A. KneissI, Montanuniversität Leoben

Dr. W.-U. Kopp, Struers GmbH, Willich

Dr. J. Paul, Leica Vertrieb GmbH, Bensheim

Prof. Dr. M. Pohl, Ruhr-Universität Bochum

Prof. Dr. W. Possart, Univ. des Saarlandes, Saarbrücken

Prof. Dr. G. Schneider, Fachhochschule Aalen

U. Taeffner, MPI für Metallforschung, Stuttgart

Dr. J. Trempler, Martin-Luther-Univ. Halle-Wittenberg

Prof. Dr. H.-H. Uchida, Tokai Univ., Hiratsuka, Kanagawa

G. Vander Voort, Buehler Ltd., Illinois

Prof. Dr. H. Vehoff, Univ. des Saarlandes, Saarbrücken

Dr. H.-J. Wieland, Verein Dt. Eisenhüttenleute, Düsseldorf

Zur Titelanzeige

Titelfoto:

Co-Basis

Superlegierung

Gußzustand

Farbätzung Beraha III

Hellfeld

Vergrößerung 200x

Günther Müller

\section{Präparation zäher Werkstoffe}

Superlegierungen, Edelstähle und ähnlich zähe Materialien neigen bei der Präparation zur Bildung von Verformungsschichten, die eine Deutung der wahren Gefügestruktur erschweren, bzw. unmöglich machen. Diese Verformungsschichten können vermieden werden, wenn die Schlußpolitur mit MASTERMET ${ }^{\circledR}$ oder FINAL durchgeführt wird, wobei dem Oxidpoliermittel

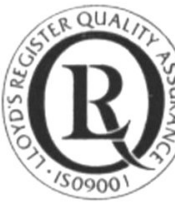

Reg.-Nr. 207017 Ergebnisse lassen sich auch mit MASTERPOLISH ${ }^{\circledR}$, einem MischoxidPoliermittel von BUEHLER, erzielen.

\section{Kompetenz in Materialographie und Werkstoffprüfung} Bei Präparationsproblemen stehen Ihnen unsere Anwendungslabors zur Verfügung

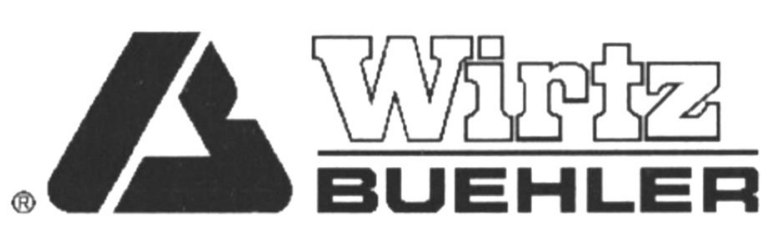

172
Wirtz-Buehler GmbH • In der Steele 2 • D-40599 Düsseldorf • Tel. 0211/974100 • Fax 0211/9741079 Wirtz-Buehler GmbH • Postfach 515 - A-8700 Leoben • Tel. 03842/46676 • Fax 03842/46678 Prüfmaschinen AG • Postfach • CH-8953 Dietikon 1 • Tel. 01/7464030 • Fax 01/7464039 email:vertrieb@wirtz-buehler.de • http://www.wirtz-buehler.de • http://www.buehlerltd.com 